CUBO A Mathematical Journal Vol.14, No 01 , (09-19). March 2012

\title{
Integral composition operators between weighted Bergman spaces and weighted Bloch type spaces
}

\author{
ELKE WOLF \\ University of Paderborn, \\ Mathematical Institute, \\ D-33095 Paderborn, Germany, \\ email: lichte@math.uni-paderborn.de
}

\begin{abstract}
We characterize boundedness and compactness of integral composition operators acting between weighted Bergman spaces $A_{v, p}$ and weighted Bloch type spaces $B_{w}$.

\section{RESUMEN}

Caracterizamos la acotación y compacidad de operadores integrales compuestos actuando entre espacios de Bergman con peso $A_{v, p}$ y espacios $B_{w}$ de tipo Bloch con peso.
\end{abstract}

Keywords and Phrases: Weighted Bergman spaces, integral composition operator, weighted Bloch type spaces

2010 AMS Mathematics Subject Classification: 47B33, 47B38. 


\section{Introduction}

Let $H(\mathbb{D})$ denote the set of all analytic functions on the open unit disk $\mathbb{D}$ of the complex plane. A map $\mathrm{g} \in \mathrm{H}(\mathbb{D})$ induces the Volterra type or Riemann-Stieltjes operator

$$
\mathrm{J}_{\mathrm{g}}: \mathrm{H}(\mathbb{D}) \rightarrow \mathrm{H}(\mathbb{D}), \mathrm{f} \mapsto \int_{0}^{z} \mathrm{f}(\xi) \mathrm{g}^{\prime}(\xi) \mathrm{d} \xi, z \in \mathbb{D} .
$$

This operator appears naturally in the study of pointwise multiplication operators since with the companion integral operator

$$
I_{g}: H(\mathbb{D}) \rightarrow H(\mathbb{D}), f \mapsto \int_{0}^{z} f^{\prime}(\xi) g(\xi) d \xi, z \in \mathbb{D}
$$

we have that

$$
J_{g} f+I_{g} f=M_{g} f-f(0) g(0)
$$

where $M_{\mathrm{g}}$ denotes the pointwise multiplication operator given by

$$
M_{g}: H(\mathbb{D}) \rightarrow H(\mathbb{D}),\left(M_{g} f\right)(z)=g(z) f(z), z \in \mathbb{D}
$$

See e.g. [1], [2], [3], [17] or [21].

Moreover, let $v$ and $w$ be strictly positive bounded and continuous functions (weights) on $\mathbb{D}$. Then the weighted Bergman space $A_{v, p}$ is defined as follows

$$
A_{v, p}=\left\{f \in H(\mathbb{D}) ;\|f\|_{v, p}:=\left(\int_{\mathbb{D}}|f(z)|^{p} v(z) d A(z)\right)^{\frac{1}{p}}<\infty\right\}, 1 \leq p<\infty
$$

where $\mathrm{d} A(z)$ is the area measure on $\mathbb{D}$ normalized so that area of $\mathbb{D}$ is 1 . Furthermore, we consider the weighted Bloch type spaces $B_{w}$ of functions $f \in H(\mathbb{D})$ satisfying $\|f\|_{B_{w}}:=\sup _{z \in \mathbb{D}} w(z)\left|f^{\prime}(z)\right|<$ $\infty$. Provided we identify functions that differ by a constant, $\|\cdot\|_{B_{w}}$ becomes a norm and $B_{w}$ a Banach space.

Let $\phi$ be an analytic self-map of $\mathbb{D}$. In [13] Li characterized boundedness and compactness of Volterra composition operators

$$
\left(J_{g, \phi} f\right)(z)=\int_{0}^{z}(f \circ \phi)(\xi)(g \circ \phi)^{\prime}(\xi) d \xi, z \in \mathbb{D},
$$

and the integral composition operators

$$
\left(I_{g, \phi} f\right)(z)=\int_{0}^{z}(f \circ \phi)^{\prime}(\xi)(g \circ \phi)(\xi) d \xi, z \in \mathbb{D},
$$

acting between weighted Bergman spaces and weighted Bloch type spaces, both generated by standard weights. In [19] we generalized his results related to the Volterra composition operators $\mathrm{J}_{\mathfrak{g}, \phi}$ 
to a more general setting. In this article our aim is to characterize boundedness and compactness of the integral composition operators $I_{g, \phi}$ acting between weighted Bergman spaces and weighted Bloch type spaces generated by a quite general class of weights.

\section{The setting}

This section is devoted to the description of the setting in which we are interested. Let $v$ be a holomorphic function on $\mathbb{D}$, non-vanishing, strictly positive on $\left[0,1\right.$ [ and satisfying $\lim _{r \rightarrow 1} v(r)=0$. Then we define the weight $v$ as follows

$$
v(z):=v\left(|z|^{2}\right) \text { for every } z \in \mathbb{D} \text {. }
$$

Next, we give some illustrating examples of weights of this type:

(i) Consider $v(z)=(1-z)^{\alpha}, \alpha>0$. Then the corresponding weight is the so-called standard weight $v(z)=\left(1-|z|^{2}\right)^{\alpha}$.

(ii) Select $v(z)=e^{-\frac{1}{(1-z)^{\alpha}}}, \alpha>0$. Then we obtain the weight $v(z)=e^{-\frac{1}{\left(1-|z|^{2}\right)^{\alpha}}}$.

(iii) Choose $v(z)=\sin (1-z)$ and the corresponding weight is given by $v(z)=\sin \left(1-|z|^{2}\right)$.

(iv) Let $v(z)=(1-\log (1-z))^{\beta}$ for some $\beta<0$. Then we get $v(z)=\left(1-\log \left(1-|z|^{2}\right)\right)^{\beta}$.

For a fixed point $a \in \mathbb{D}$ we introduce a function $v_{a}(z):=v(\bar{a} z)$ for every $z \in \mathbb{D}$. Since $v$ is holomorphic on $\mathbb{D}$, so is the function $v_{\mathrm{a}}$.

We say that a weight $v$ is radial if $v(z)=v(|z|)$ for every $z \in \mathbb{D}$. Moreover, radial weights are typical if additionally $\lim _{|z| \rightarrow 1} v(z)=0$ holds. Thus, we introduced a class of typical weights. In [15] Lusky studied weights satisfying the following condition (L1) which was renamed after the author:

$$
\text { (L1) } \inf _{n \in \mathbb{N}} \frac{v\left(1-2^{-n-1}\right)}{v\left(1-2^{-n}\right)}>0 .
$$

Among others examples of weights satisfying condition (L1) are the standard weights (see Example (i)) and the logarithmic weights (Example (iv)). Throughout this work condition (L1) will play a great role, and we will need the following condition $(A)$ which is equivalent to (L1):

(A) there are $0<\mathrm{r}<1$ and $1<\mathrm{C}<\infty$ with $\frac{v(z)}{v(\mathrm{p})} \leq \mathrm{C}$ for all $\mathrm{p}, z \in \mathbb{D}$ with $\rho(\mathrm{p}, z) \leq \mathrm{r}$.

The equivalence of the conditions (L1) and (A) was shown in [10]. See also [14]. 


\section{Basic facts}

We need some geometric data of the open unit disk. Fix $a \in \mathbb{D}$ and consider the authomorphism $\varphi_{\mathrm{a}}(z):=\frac{z-\mathrm{a}}{1-\overline{\mathrm{a}} z}, z \in \mathbb{D}$, which interchanges 0 and $\mathrm{a}$. Moreover, we use the fact that

$$
\varphi_{\mathrm{a}}^{\prime}(z)=\frac{|\mathrm{a}|^{2}-1}{(1-\overline{\mathrm{a}} z)^{2}}, z \in \mathbb{D} .
$$

Now, the pseudohyperbolic metric is given by

$$
\rho(z, a)=\left|\varphi_{\mathrm{a}}(z)\right|, z, a \in \mathbb{D} \text {. }
$$

One of the most important properties of the pseudohyperbolic metric is that it is Möbius invariant, that is,

$$
\rho(\sigma(z), \sigma(a))=\rho(z, a) \text { for every automorphism } \sigma \text { of } \mathbb{D}, z, a \in \mathbb{D} \text {. }
$$

The pseudohyperbolic metric is a true metric. In fact, it even satisfies a stronger version of the triangle inequality, more precisely, for every $z, a, b \in \mathbb{D}$ we have that

$$
\rho(z, a) \leq \frac{\rho(z, b)+\rho(b, a)}{1+\rho(z, b) \rho(b, a)} .
$$

\section{Results}

Before we are able to treat boundedness and compactness of operators $I_{\mathfrak{g}, \phi}$ we need a number of auxiliary lemmas. The first lemma is taken from [18].

Lemma 1. Let $v$ be a weight as defined in (2.1) such that $\sup _{\mathrm{a} \in \mathbb{D}} \sup _{z \in \mathbb{D}} \frac{v(z)\left|v_{\mathrm{a}}\left(\varphi_{\mathrm{a}}(z)\right)\right|}{v\left(\varphi_{\mathrm{a}}(z)\right)} \leq \mathrm{C}<\infty$. Then

$$
|f(z)| \leq \frac{C^{\frac{1}{p}}}{v(0)^{\frac{1}{p}}\left(1-|z|^{2}\right)^{\frac{2}{p}} v(z)^{\frac{1}{p}}}\|f\|_{\nu, p}
$$

for all $z \in \mathbb{D}, f \in A_{v, p}$.

Calculations show that the examples (i) -(iv) which were listed up above satisfy the assumptions of the previous lemma. The next lemma was shown in [20].

Lemma 2. Let $v$ be a radial weight as defined in (2.1) such that $v$ additionally satisfies condition (L1). Then for every $\mathrm{f} \in \mathrm{A}_{v, \mathrm{p}}$ there is $\mathrm{C}_{v}>0$ such that

$$
|f(z)-f(w)| \leq C_{v}\|f\|_{v, p} \max \left\{\frac{1}{\left(1-|z|^{2}\right)^{\frac{2}{p}} v(z)^{\frac{1}{p}}}, \frac{1}{\left(1-|w|^{2}\right)^{\frac{2}{p}} v(w)^{\frac{1}{p}}}\right\} \rho(z, w)
$$

for every $z, w \in \mathbb{D}$. 
Lemma 3. Let $v$ be a radial weight as defined in (2.1) such that $v$ additionally satisfies condition (L1) and $\sup _{\mathbf{a} \in \mathbb{D}} \sup _{z \in \mathbb{D}} \frac{v(z)\left|v_{a}\left(\varphi_{a}(z)\right)\right|}{v\left(\varphi_{a}(z)\right)} \leq \mathrm{C}<\infty$. Then

$$
\left|f^{\prime}(z)\right| \leq \frac{C^{\frac{1}{p}}}{v(0)^{\frac{1}{p}}\left(1-|z|^{2}\right)^{\frac{2}{p}+1} v(z)^{\frac{1}{p}}}\|\mathbf{f}\|_{\nu, p}
$$

for every $z \in \mathbb{D}$ and every $\mathrm{f} \in \mathrm{A}_{v, \mathrm{p}}$.

Proof. Lemma 2 yields that for every $\mathrm{f} \in \mathrm{A}_{v, \mathrm{p}}$ and every $\mathrm{h}, z \in \mathbb{D}$ with $z+\mathrm{h} \in \mathbb{D}$, we have

$$
|f(z+h)-f(z)| \leq C_{v}\|f\|_{v, p} \max \left\{\frac{1}{\left(1-|z+h|^{2}\right)^{\frac{2}{p}} v(z+h)^{\frac{1}{p}}}, \frac{1}{\left(1-|z|^{2}\right)^{\frac{2}{p}} v(z)^{\frac{1}{p}}}\right\} \frac{|h|}{|1-\bar{z}(z+h)|} .
$$

Hence

$$
\left|\frac{f(z+h)-f(z)}{h}\right| \leq C_{v}\|f\|_{v, p} \max \left\{\frac{1}{\left(1-|z+h|^{2}\right)^{\frac{2}{p}} v(z+h)^{\frac{1}{p}}}, \frac{1}{\left(1-|z|^{2}\right)^{\frac{2}{p}} v(z)^{\frac{1}{p}}}\right\} \frac{1}{|1-\bar{z}(z+h)|}
$$

and finally

$$
\begin{aligned}
\left|f^{\prime}(z)\right| & =\left|\lim _{h \rightarrow 0} \frac{f(z+h)-f(z)}{h}\right| \\
& \leq \lim _{h \rightarrow 0} C_{v}\|f\|_{v, p} \max \left\{\frac{1}{\left(1-|z+h|^{2}\right)^{\frac{2}{p}} v(z+h)^{\frac{1}{p}}}, \frac{1}{\left(1-|z|^{2}\right)^{\frac{2}{p}} v(z)^{\frac{1}{p}}}\right\} \frac{1}{|1-\bar{z}(z+h)|} \\
& =C_{v}\|f\|_{v, p} \frac{1}{\left(1-|z|^{2}\right)^{\frac{2}{p}+1} v(z)^{\frac{1}{p}}}
\end{aligned}
$$

for every $z \in \mathbb{D}$, as desired.

Lemma 4. Let $v$ be a radial weight as in Lemma 3. Then there exist $0<\mathrm{r}<1$ and a constant $M>0$ such that for $\mathrm{f} \in \mathrm{A}_{v, \mathrm{p}}$

$$
\left|f^{\prime}(z)-f^{\prime}(w)\right| \leq \frac{4 M C^{\frac{1}{p}}}{v(0)^{\frac{1}{p}}} \frac{\|f\|_{v, p}}{r\left(1-|z|^{2}\right)^{\frac{2}{p}+1} v(z)^{\frac{1}{p}}} \rho(z, w)
$$

for every $z, w \in \mathbb{D}$ with $\rho(z, w) \leq \frac{r}{2}$.

Proof. By hypotesis, $v$ has condition (L1), and, moreover, we know that (L1) is equivalent to condition (A). Since the weight $u(z)=1-|z|^{2}$ also satisfies condition (L1), we can find $0<r<1$ and constants $M_{1}<\infty$ and $M_{2}<\infty$ such that

$$
\frac{v(z)}{v(w)} \leq M_{1} \text { and } \frac{1-|z|^{2}}{1-|w|^{2}} \leq M_{2} \text { for every } z, w \in \mathbb{D} \text { with } \rho(z, w) \leq r .
$$

Let $w \in \mathbb{D}$ be fixed. Since

$$
\varphi_{w}\left(\varphi_{w}(z)\right)=z \text { and } \varphi_{w}(0)=w,
$$


we get that

$$
\left|f^{\prime}(z)-f^{\prime}(w)\right|=\mid f^{\prime}\left(\varphi_{w}\left(\varphi_{w}(z)\right)-f^{\prime}\left(\varphi_{w}\left(\varphi_{w}(w)\right) \mid .\right.\right.
$$

For $|z|=\rho\left(\varphi_{w}(z), w\right) \leq r$ we obtain by using Lemma 3

$$
\begin{aligned}
\left|f^{\prime}\left(\varphi_{w}(z)\right)\right| & \leq \frac{C^{\frac{1}{p}}\|f\|_{v, p}}{v(0)^{\frac{1}{p}}\left(1-\left|\varphi_{w}(z)\right|^{2}\right)^{\frac{2}{p}+1} v\left(\varphi_{w}(z)\right)^{\frac{1}{p}}} \\
& =\frac{C^{\frac{1}{p}}\|f\|_{v, p}}{v(0)^{\frac{1}{p}}\left(1-|w|^{2}\right)^{\frac{2}{p}+1} v(w)^{\frac{1}{p}}} \frac{\left(1-|w|^{2}\right)^{\frac{2}{p}+1} v(w)^{\frac{1}{p}}}{\left(1-\left|\varphi_{w}(z)\right|^{2}\right)^{\frac{2}{p}+1} v\left(\varphi_{w}(z)\right)^{\frac{1}{p}}} \\
& \leq \frac{C^{\frac{1}{p}} M_{1}^{\frac{1}{p}} M_{2}^{\frac{2}{p}+1}}{v(0)^{\frac{1}{p}}} \frac{\|f\|_{v, p}}{\left(1-|w|^{2}\right)^{\frac{2}{p}+1} v(w)^{\frac{1}{p}}} .
\end{aligned}
$$

Let us now consider $g_{w}(z):=f^{\prime}\left(\varphi_{w}(z)\right)$ for every $z \in \mathbb{D}$. Thus, for $\rho(z, w)=\left|\varphi_{w}(z)\right| \leq \frac{r}{2}$ we can find $\Theta \in \mathbb{D}$ with $|\Theta| \leq\left|\varphi_{w}(z)\right| \leq \frac{r}{2}$ such that

$$
\begin{aligned}
\left|f^{\prime}(z)-f^{\prime}(w)\right| & =\left|g_{w}\left(\varphi_{w}(z)\right)-g_{w}(0)\right| \\
& \leq\left|\varphi_{w}(z)\right|\left|\int_{0}^{1}\left[\frac{\partial}{\partial t} g_{w}\right]\left(t \varphi_{w}(z)\right) d t\right| \\
& \leq\left|\varphi_{w}(z)\right|\left|\frac{\partial}{\partial z} g_{w}(\Theta)\right| \\
& =\left|\varphi_{w}(z)\right| \frac{1}{2 \pi}\left|\int_{|\xi|=r} \frac{g_{w}(\xi)}{(\xi-\Theta)^{2}} d \Theta\right|
\end{aligned}
$$

Finally,

$$
\begin{array}{r}
\left|f^{\prime}(z)-f^{\prime}(w)\right| \leq \frac{C^{\frac{1}{p}} M_{1}^{\frac{1}{p}} M_{2}^{\frac{2}{p}+1}}{v(0)^{\frac{1}{p}}} \frac{\left|\varphi_{w}(z)\right| r\|f\|_{v, p}}{\left(r-\left|\varphi_{w}(z)\right|\right)^{2}\left(1-|w|^{2}\right)^{\frac{2}{p}+1} v(w)^{\frac{1}{p}}} \\
\leq \frac{4 C^{\frac{1}{p}} M_{1}^{\frac{1}{p}} M_{2}^{\frac{2}{p}+1}}{v(0)^{\frac{1}{p}}} \frac{\rho(z, w)\|f\|_{v, p}}{r\left(1-|w|^{2}\right)^{\frac{2}{p}+1} v(w)^{\frac{1}{p}}} .
\end{array}
$$

We select $M:=M_{1}^{\frac{1}{p}} M_{2}^{\frac{2}{p}+1}$ and obtain the claim.

Lemma 5. Let $v$ be a weight as in Lemma 3. Then, there is $C_{v}>0$ such that for every $f \in A_{v, p}$

$$
\left|f^{\prime}(z)-f^{\prime}(w)\right| \leq C_{v}\|f\|_{v, p} \max \left\{\frac{1}{\left(1-|z|^{2}\right)^{\frac{2}{p}+1} v(z)^{\frac{1}{p}}}, \frac{1}{\left(1-|w|^{2}\right)^{\frac{2}{p}+1} v(w)^{\frac{1}{p}}}\right\} \rho(z, w)
$$

for every $z, w \in \mathbb{D}$.

Proof. By Lemma 4 we can find $0<s<1$ and a constant $M<\infty$ such that

$$
\left|f^{\prime}(z)-f^{\prime}(w)\right| \leq \frac{4 M C^{\frac{1}{p}}}{v(0)^{\frac{1}{p}}} \frac{\|f\|_{v, p}}{s\left(1-|z|^{2}\right)^{\frac{2}{p}+1} v(z)^{\frac{1}{p}}} \rho(z, w)
$$


for every $z, w \in \mathbb{D}$ with $\rho(z, w) \leq \frac{s}{2}$. Next, if $\rho(z, w)>\frac{s}{2}$, then

$$
\begin{aligned}
\mid f^{\prime}(z) & -f^{\prime}(w) \mid \leq 2 \frac{C^{\frac{1}{p}}}{v(0)^{\frac{1}{p}}}\|f\|_{v, p} \max \left\{\frac{1}{\left(1-|z|^{2}\right)^{\frac{2}{p}+1} v(z)^{\frac{1}{p}}}, \frac{1}{\left(1-|w|^{2}\right)^{\frac{2}{p}+1} v(w)^{\frac{1}{p}}}\right\} \\
& \leq \frac{4}{s} \frac{C^{\frac{1}{p}}}{v(0)^{\frac{1}{p}}}\|f\|_{v, p} \max \left\{\frac{1}{\left(1-|z|^{2}\right)^{\frac{2}{p}+1} v(z)^{\frac{1}{p}}}, \frac{1}{\left(1-|w|^{2}\right)^{\frac{2}{p}+1} v(w)^{\frac{1}{p}}}\right\} \rho(z, w) .
\end{aligned}
$$

Hence, with $C_{v}:=\max \left\{\frac{4 M C^{\frac{1}{p}}}{v(0)^{\frac{1}{p}} s}, \frac{4 C^{\frac{1}{p}}}{s v(0)^{\frac{1}{p}}}\right\}$, we conclude

$$
\left|f^{\prime}(z)-f^{\prime}(w)\right| \leq C_{v} \max \left\{\frac{1}{\left(1-|z|^{2}\right)^{\frac{2}{p}+1} v(z)^{\frac{1}{p}}}, \frac{1}{\left(1-|w|^{2}\right)^{\frac{2}{p}+1} v(w)^{\frac{1}{p}}}\right\} \rho(z, w)
$$

for every $z, w \in \mathbb{D}$ and the claim follows.

Inductively, we can show the following lemmas:

Lemma 6. Let $v$ be a weight as in Lemma 3. Then there is $C_{v}>0$ such that for every $\mathrm{f} \in A_{v, p}$

$$
\left|f^{(n)}(z)\right| \leq \frac{C_{v}}{\left(1-|z|^{2}\right)^{\frac{2}{p}+n} v(z)^{\frac{1}{p}}}\|f\|_{v, p}
$$

for every $z \in \mathbb{D}$ and every $\mathrm{n} \in \mathbb{N}_{0}$.

Lemma 7. Let $v$ be a weight as in Lemma 3. Then there exists $C_{v}>0$ such that for every $\mathrm{f} \in \mathrm{A}_{v, \mathrm{p}}$

$$
\left|f^{(n)}(z)-f^{(n)}(w)\right| \leq C_{v}\|f\|_{v, p} \max \left\{\frac{1}{\left(1-|z|^{2}\right)^{\frac{2}{p}+n} v(z)^{\frac{1}{p}}}, \frac{1}{\left(1-|w|^{2}\right)^{\frac{2}{p}+n} v(w)^{\frac{1}{p}}}\right\} \rho(z, w)
$$

for every $z, w \in \mathbb{D}$ and every $\mathrm{n} \in \mathbb{N}_{0}$.

Now, we turn our attention to the operators $I_{g, \phi}$ and start with characterizing when they are bounded.

Theorem 8. Let $w$ be a weight and $v$ be a weight as in Lemma 3 with $M:=\sup _{\mathbf{a} \in \mathbb{D}} \sup _{z \in \mathbb{D}} \frac{v(z)}{|v(\overline{\mathbf{a}} z)|}<$ $\infty$. If

$$
\sup _{z \in \mathbb{D}} \frac{w(z)\left|\phi^{\prime}(z) g(\phi(z))\right|}{\left(1-|\phi(z)|^{2}\right)^{\frac{2}{p}+1} v(\phi(z))^{\frac{1}{p}}}<\infty
$$

then the operator $\mathrm{I}_{\mathrm{g}, \Phi}: \mathrm{A}_{v, \mathrm{p}} \rightarrow \mathrm{B}_{w}$ is bounded. If we assume additionally that

$$
\sup _{z \in \mathbb{D}} \frac{\left|v^{\prime}\left(|\phi(z)|^{2}\right)\right| w(z)\left|\phi^{\prime}(z) g(\phi(z))\right|}{v(\phi(z))^{\frac{1}{p}+1}\left(1-|\phi(z)|^{2}\right)^{\frac{2}{p}}}<\infty
$$

then the converse is also true. 
Proof. We start with assuming that the operator $\mathrm{I}_{\mathrm{g}, \phi}$ is bounded and that the condition (4.2) is satisfied. Fix a point $a \in \mathbb{D}$ and set

$$
f_{a}(z):=\frac{\varphi_{a}^{\prime}(z)^{\frac{2}{p}}}{v(\bar{a} z)^{\frac{1}{p}}} \text { for every } z \in \mathbb{D}
$$

Then

$$
\|f\|_{\nu, p}^{p}=\int_{\mathbb{D}} \frac{\left|\varphi_{\mathrm{a}}^{\prime}(z)\right|^{2}}{|v(\overline{\mathrm{a}} z)|} v(z) \mathrm{dA}(z) \leq \sup _{z \in \mathbb{D}} \frac{v(z)}{|v(\overline{\mathrm{a}} z)|} \int_{\mathbb{D}}\left|\varphi_{\mathrm{a}}^{\prime}(z)\right|^{2} \mathrm{~d} A(z) \leq \sup _{z \in \mathbb{D}} \frac{v(z)}{|v(\overline{\mathrm{a}} z)|} \leq M
$$

and the constant $M$ is independent of the choice of the point $a$. For the derivative we have

$$
f_{a}^{\prime}(z)=\frac{2}{p} \frac{\varphi_{a}^{\prime}(z)^{\frac{2}{p}-1} \varphi_{a}^{\prime \prime}(z)}{v(\bar{a} z)^{\frac{1}{p}}}-\frac{1}{p} \frac{\bar{a} v^{\prime}(\bar{a} z) \varphi_{a}^{\prime}(z)^{\frac{2}{p}}}{v(\bar{a} z)^{\frac{1}{p}+1}}
$$

for every $z \in \mathbb{D}$. Hence we can find a constant $C^{*}>0$ such that

$$
\begin{aligned}
\left|\frac{w(a)\left|\phi^{\prime}(a) \| g(\phi(a))\right|}{\left(1-|\phi(a)|^{2}\right)^{\frac{2}{p}+1} v(\phi(a))^{\frac{1}{p}}}-\frac{\left|v^{\prime}\left(|\phi(a)|^{2}\right)\right| w(a)\left|\phi^{\prime}(a) g(\phi(a))\right|}{v(\phi(a))^{\frac{1}{p}+1}\left(1-|\phi(a)|^{2}\right)^{\frac{2}{p}}}\right| \leq\left|f_{\phi(a)}^{\prime}(\phi(a))\right| w(a)\left|g(\phi(a)) \| \phi^{\prime}(a)\right| \mid \\
\leq\left|\left(I_{g, \phi} f_{\phi(a)}\right)^{\prime}(a)\right| w(a) \\
\leq C^{*}\left\|J_{g, \phi}\right\|\left\|f_{\phi(a)}\right\|_{v, p}
\end{aligned}
$$

Finally, since (4.2) is fulfilled and the operator $\mathrm{I}_{\mathrm{g}, \phi}$ is bounded, the claim follows. Conversely, an application of Lemma 3 yields for $f \in A_{v, p}$

$$
\begin{aligned}
\sup _{z \in \mathbb{D}}\left|\left(I_{g, \phi} f\right)^{\prime}(z)\right| w(z) & =\sup _{z \in \mathbb{D}}\left|f^{\prime}(\phi(z))\|g(\phi(z))\| \phi^{\prime}(z)\right| w(z) \\
& \leq \sup _{z \in \mathbb{D}} \frac{C^{\frac{1}{p}}\|f\|_{v, p} w(z)\left|g(\phi(z)) \| \phi^{\prime}(z)\right|}{v\left(1-\left.\phi(z)\right|^{2}\right)^{\frac{2}{p}+1} v(\phi(z))^{\frac{1}{p}}} .
\end{aligned}
$$

Hence the claim follows.

Next, we study, when such operators are compact. To do this we need a lemma which can easily be derived from [9] Proposition 3.11.

Lemma 9. Let $v$ and $w$ be weights. Then the operator $\mathrm{I}_{\mathrm{g}, \phi}: \mathrm{A}_{v, \mathrm{p}} \rightarrow \mathrm{B}_{w}$ is compact if and only if it is bounded and for every bounded sequence $\left(\mathrm{f}_{\mathrm{n}}\right)_{\mathrm{n}}$ in $\mathrm{A}_{v, \mathrm{p}}$ which converges to zero uniformly on the compact subsets of $\mathbb{D}, \mathrm{I}_{\mathrm{g}, \phi} \mathrm{f}_{\mathrm{n}}$ tends to zero in $\mathrm{B}_{w}$ if $\mathrm{n} \rightarrow \infty$.

Theorem 10. Let $w$ be a weight and $v$ be a weight as in Theorem 8. Moreover, we assume that $\mathrm{I}_{\mathrm{g}, \phi}: A_{v, \mathrm{p}} \rightarrow \mathrm{B}_{w}$ is bounded. If

$$
\lim _{r \rightarrow 1} \sup _{|\phi(z)|>r} \frac{w(z)\left|\phi^{\prime}(z) g(\phi(z))\right|}{\left(1-|\phi(z)|^{2}\right)^{\frac{2}{p}+1} v(\phi(z))^{\frac{1}{p}}}=0
$$


then the operator $\mathrm{I}_{\mathrm{g}, \Phi}: \mathrm{A}_{v, \mathrm{p}} \rightarrow \mathrm{B}_{w}$ is compact. If we assume additionally

$$
\lim _{r \rightarrow 1} \sup _{|\phi(z)|>r} \frac{\left|v^{\prime}\left(|\phi(z)|^{2}\right)\right| w(z)\left|\phi^{\prime}(z) g(\phi(z))\right|}{v(\phi(z))^{\frac{1}{p}+1}\left(1-|\phi(z)|^{2}\right)^{\frac{2}{p}}}=0
$$

then the converse is also true.

Proof. Assume that the operator $\mathrm{I}_{g, \phi}: \mathrm{A}_{v, p} \rightarrow \mathrm{B}_{w}$ is compact and that (4.4) is satisfied. To show (4.3) let $\left(z_{\mathfrak{n}}\right)_{\mathfrak{n}}$ be a sequence with $\left|\phi\left(z_{\mathfrak{n}}\right)\right| \rightarrow 1$ and put

$$
f_{k}(z):=\frac{\varphi_{\phi\left(z_{k}\right)}^{\prime}(z)^{\frac{2}{p}}}{v\left(\overline{\phi\left(z_{k}\right)} z\right)^{\frac{1}{p}}} \text { for every } z \in \mathbb{D} \text { and every } k \in \mathbb{N}
$$

Analogously to the proof of Theorem 8 we can show that $\left(f_{n}\right)_{n}$ is a bounded sequence which tends to zero uniformly on the compact subsets of $\mathbb{D}$. Since $I_{g, \phi}$ is compact, by Lemma 9

$$
\left\|\mathrm{I}_{\mathrm{g}, \phi} \mathrm{f}_{\mathrm{n}}\right\|_{\mathrm{B}_{w}} \rightarrow 0 \text { if } \mathrm{n} \rightarrow \infty \text {. }
$$

Thus,

$$
\left\|\mathrm{I}_{g, \phi} f_{n}\right\|_{B_{w}} \geq\left|\frac{w\left(z_{n}\right) \mid \phi^{\prime}\left(z_{n}\right) \| g\left(\phi\left(z_{n}\right) \mid\right.}{\left(1-\left|\phi\left(z_{n}\right)\right|^{2}\right)^{\frac{2}{p}+1} v\left(\phi\left(z_{n}\right)\right)^{\frac{1}{p}}}-\frac{\left|v^{\prime}\left(\left|\phi\left(z_{n}\right)\right|^{2}\right)\right| w\left(z_{n}\right)\left|\phi^{\prime}\left(z_{n}\right) g\left(\phi\left(z_{n}\right)\right)\right|}{v\left(\phi\left(z_{n}\right)\right)^{\frac{1}{p}+1}\left(1-\left|\phi\left(z_{n}\right)\right|^{2}\right)^{\frac{2}{p}}}\right|,
$$

and, since (4.4) holds, condition (4.3) follows.

Conversely, suppose that (4.3) is satisfied. Let $\left(f_{n}\right)_{n}$ be a bounded sequence in $A_{v, p}$ such that $\left\|f_{n}\right\|_{\nu, p} \leq M_{1}<\infty$ for every $n \in \mathbb{N}$ and such that $\left(f_{n}\right)_{n}$ converges uniformly to zero on the compact subsets of $\mathbb{D}$ if $n \rightarrow \infty$. For a fixed $\varepsilon>0$ we can find $0<r_{0}<1$ such that if $|\phi(z)|>r_{0}$, then

$$
\frac{w(z)|g(\phi(z))|\left|\phi^{\prime}(z)\right|}{\left(1-|\phi(z)|^{2}\right)^{\frac{2}{p}+1} v(\phi(z))^{\frac{1}{p}}}<\frac{\varepsilon v(0)^{\frac{1}{p}}}{2 C^{\frac{1}{p}} M_{1}} .
$$

Moreover, we can find $M_{2}>0$ such that

$$
\sup _{|\phi(z)| \leq r_{0}} w(z)\left|g(\phi(z)) \| \phi^{\prime}(z)\right| \leq M_{2}
$$

There is $n_{0} \in \mathbb{N}$ such that

$$
\sup _{|\phi(z)| \leq \mathrm{r}_{0}}\left|\mathrm{f}_{n}^{\prime}(\phi(z))\right| \leq \frac{\varepsilon}{2 M_{2}} \text { for every } \mathrm{n} \geq \mathrm{n}_{0}
$$


We obtain applying Lemma 3

$$
\begin{aligned}
\sup _{z \in \mathbb{D}}\left|\left(I_{g, \phi} f_{\mathfrak{n}}\right)^{\prime}(z)\right| w(z) & =\sup _{z \in \mathbb{D}} w(z)\left|f_{n}^{\prime}(\phi(z)) \| g(\phi(z))\right|\left|\phi^{\prime}(z)\right| \\
& \leq \sup _{|\phi(z)| \leq r_{0}} w(z)\left|f_{\mathfrak{n}}^{\prime}(\phi(z))\|g(\phi(z))\| \phi^{\prime}(z)\right| \\
& +\sup _{|\phi(z)|>r_{0}} w(z)\left|f_{n}^{\prime}(\phi(z))\right||g(\phi(z))|\left|\phi^{\prime}(z)\right| \\
& \leq \sup _{|\phi(z)| \leq r_{0}}\left|f_{n}^{\prime}(\phi(z))\right| \sup _{|\phi(z)| \leq r_{0}} w(z)|g(\phi(z))|\left|\phi^{\prime}(z)\right| \\
& +\sup _{|\phi(z)|>r_{0}} \frac{C^{\frac{1}{p}}\left\|f_{n}\right\|\left\|_{v, p} w(z)\left|g(\phi(z)) \| \phi^{\prime}(z)\right|\right.}{\left.1-|\phi(z)|^{2}\right)^{\frac{2}{p}+1} v(\phi(z))^{\frac{1}{p}}} \\
& \leq \varepsilon,
\end{aligned}
$$

and the claim follows.

Received: March 2011. Revised: April 2011.

\section{References}

[1] A. Aleman, J.A. Cima, An integral operator on $\mathrm{H}^{p}$ and Hardy's inequality, J. Anal. Math. 85 (2001), 157-176

[2] A. Aleman, A. G. Siskakis, An integral operator on $\mathrm{H}^{\mathrm{p}}$, Complex Variables Theory Appl. 28 (1995), no. 2, 149- 158.

[3] A. Aleman, A. G. Siskakis, Integration operators on Bergman spaces, Indiana University Math. J. 46 (1997), no. 2, 337-356.

[4] J. Bonet, P. Domański, M. Lindström, Essential norm and weak compactness of composition operators on weighted Banach spaces of analytic functions, Canad. Math. Bull. 42, no. 2, (1999), 139-148.

[5] J. Bonet, P. Domański, M. Lindström, J. Taskinen, Composition operators between weighted Banach spaces of analytic functions, J. Austral. Math. Soc. (Serie A) 64 (1998), 101-118.

[6] J. Bonet, M. Friz, E. Jordá, Composition operators between weighted inductive limits of spaces of holomorphic functions, Publ. Math. 67 (2005), no. 3-4, 333-348.

[7] J. Bonet, M. Lindström, E. Wolf, Differences of composition operators between weighted Banach spaces of holomorphic functions, to appear in J. Austr. Math. Soc.

[8] M.D. Contreras, A.G. Hernández-Díaz, Weighted composition operators in weighted Banach spaces of analytic functions, J. Austral. Math. Soc. (Serie A) 69 (2000), 41-60. 
[9] C. Cowen, B. MacCluer, Composition Operators on Spaces of Analytic Functions, CRC Press, Boca Raton, 1995.

[10] P. Domański, M. Lindström, Sets of interpolation and sampling for weighted Banach spaces of holomorphic functions, Ann. Pol. Math (2002) 79, 233-264.

[11] P. Duren, A. Schuster, Bergman spaces, Mathematical Surveys and Monographs 100, American Mathematical Society, Providence, RI, 2004.

[12] H. Hedenmalm, B. Korenblum, K. Zhu, Theory of Bergman spaces, Graduate Texts in Mathematics 199, Springer-Verlag, New York, 2000.

[13] S. Li, Volterra composition operators between weighted Bergman spaces and Bloch type spaces, J. Korean Math. Soc. 45(2008), no. 1, 229-248.

[14] M. Lindström, E. Wolf, Essential norm of the difference of weighted composition operators, Monatsh. Math (2008) 153, 133-143.

[15] W. Lusky, On the structure of $\mathrm{H} v_{0}$ (D) and $h v_{0}$ (D), Math. Nachr. 159 (1992), 279-289.

[16] J.H. Shapiro, Composition Operators and Classical Function Theory, Springer, 1993.

[17] A. G. Sisakis, R. Zhao, A Volterra type oprator on spaces of analytic functions, Functions spaces (Edwardville IL, 1998), 299-311, Contemp. Math. 232, Amer. Math. Soc. Providence, RI, 1999.

[18] E. Wolf, Weighted composition operators between weighted Bergman spaces, RACSAM Rev. R. Acad. Cien. Serie A Mat. 103, no. 1, 11-15.

[19] E. Wolf, Volterra composition operators between weighted Bergman spaces and weighted Bloch type spaces, Collect Math. 61 (2010), no. 1, 57-63.

[20] E. Wolf, Differences of weighted compostiion operators between Bergman spaces and weighted Banach spaces of holomorphic functions, Glasgow Math. J. 52 (2010), 325-332.

[21] J. Xiao, Riemann-Stieltjes operators on weighted Bloch and Bergman spaces of the unit ball, J. London, Math. Soc. (2), 70 (2004), no. 1, 199-214. 